\title{
Comparative Evaluation of Discomfort, Expectations and Functional Experiences during Treatment of Class II Malocclusion with Forsus Fixed Functional Appliance and Sharma's Class II Corrector - A Questionnaire Based Survey
}

\author{
Shriya Prakash Murarka ${ }^{1}$, Sunita Shrivastav², Ranjit Kamble ${ }^{3}$, Hamza Dargahwala ${ }^{4}$, \\ Prutha Khakhar ${ }^{5}$, Zynul John ${ }^{6}$, Purva Dhannawat ${ }^{7}$, Shruti Rathi ${ }^{8}$ \\ 1, 2, 3, 4, 5, 6, 7, 8 Department of Orthodontics and Dentofacial Orthopaedics, \\ Sharad Pawar Dental College, Sawangi, Meghe, Wardha, Maharashtra, India.
}

\section{ABSTRACT}

\section{BACKGROUND}

The Forsus fatigue resistant device (FFRD) appliance is known to correct Class II malocclusion. The disadvantage of it are labial flaring of lower incisors, distalisation and extrusion of maxillary molars, difficulty in procuring the appliances in remote areas and cost. No research has documented the comparison of patient's experience with FFRD and Sharma's Class II corrector appliance. Therefore, a questionnaire survey was conducted.

\section{METHODS}

40 patients having Class II Division 1 malocclusion were included and were divided into two groups- FFRD appliance (group 1, 20 patients) and Sharma's Class II corrector (group 2, 20 patients). A questionnaire was framed that consisted of 15 questions. Descriptive and analytical statistics was done using SPSS software. The difference in proportions was calculated by chi-square test. The level of significance was set at $\mathrm{P}<0.05$.

\section{RESULTS}

$30 \%$ of cases in group 2 indicated that the Sharma's Class II corrector looks good (Q1) as compared to $15 \%$ in group $1 .(P=0.630) 5 \%$ indicated it was not aesthetic in group 2 as compared to $10 \%$ in group $1.30 \%$ of cases in group 1 indicated that there were problems associated with speech as compared to $0 \%$ in group 2 . ( $\mathrm{P}<$ 0.05). Values were statistically significant.

\section{CONCLUSIONS}

Sharma's Class II corrector has similar patient acceptance as compared to FFRD appliance with the additional benefit of cost effectiveness. Hence, this can be considered as a better option in treating Class II malocclusion with fixed therapy.

\section{KEY WORDS}

FFRD, Fixed Function Appliance, Economic Orthodontics, Growth Modification, Sharma's Class II Corrector
Corresponding Author: Dr. Shriya Prakash Murarka, Murarka Garden Near Old Arvi Stand Wardha, Maharashtra, India. E-mail: shriya.m13@gmail.com

DOI: 10.14260/jemds/2021/104

How to Cite This Article:

Murarka SP, Shrivastav S, Kamble R, et al. Comparative evaluation of discomfort, expectations and functional experiences during treatment of class ii malocclusion with forsus fixed functional appliance and sharma's class ii corrector - a questionnaire-based survey. J Evolution Med Dent Sci 2021;10(08):474-478, DOI: 10.14260/jemds/2021/104

Submission 15-09-2020,

Peer Review 11-12-2020,

Acceptance 18-12-2020,

Published 22-02-2021.

Copyright (C) 2021 Shriya Prakash Murarka et al. This is an open access article distributed under Creative Commons Attribution License [Attribution 4.0 International (CC BY 4.0)] 


\section{BACKGROUND}

"Skeletal Class II Division 1 malocclusion is an anteroposterior discrepancy of maxilla and mandible which often results from either maxillary prognathism, or mandibular retrognathism or a combination of both." In cases of developing Class II Division 1, with functional retrusion of the lower jaw, ${ }^{1}$ the treatment can be done either by using removable myofunctional appliance (RMA) ${ }^{2,3}$ or fixed functional appliances (FFA) in non-compliant cases.

Also, in a Class II division 1 case with only residual growth spurt remaining, FFA are recommended. FFA are preferred over RMA, as patient compliance during growth modulation therapy can influence the treatment outcome with only limited growth remaining. The Forsus fatigue resistant device appliance was developed by William Vogt in 2006 to correct Class II malocclusion and has gained more popularity. It's a prefabricated intermaxillary push spring appliance which has an advantage of being used along with comprehensive fixed appliance therapy.4 The disadvantage of this appliance like any other fixed functional appliance include: labial flaring of lower incisors, distalisation and extrusion of maxillary molars apart from difficulty in procuring the appliances in remote area and lastly the cost. Also, if any part of the appliance breaks or is lost then procurement of new parts or appliance may pose a risk of delay in the treatment and to a certain extent relapse may occur. ${ }^{4}$

Sharma's Class II corrector is a type of FFA, developed and patented by Dr. Narendra Sharma in $2014 . .^{5}$ It is a custommade, hygienic, stable on fixation, easy to fabricate and economical for the rural set up. The chair side adjustments are possible according to the patient's requirements in the appliance dimension, length and force level can also be altered easily. ${ }^{5}$

There are multiple articles and meta-analysis that have established the efficacy of Forsus fixed functional appliance and its role as a fixed Class II corrector and patient's experience with FFRD. However, it has certain limitations such as its high cost and frequent breakages of the appliance. Therefore, Sharma's Class II corrector appliance was developed in the department to overcome the drawbacks associated with Forsus fixed functional appliance and has been in use ever since. There are studies published related to the skeletal and dental changes associated with Sharma's Class II corrector. However, no research has documented the comparison of patient's experience with FFRD and Sharma's Class II corrector appliance. Therefore, a questionnaire survey was conducted to evaluate discomfort, expectations and experiences of patients' during treatment of Class II malocclusion with Forsus fixed functional appliance with those observed in Sharma's Class II corrector.

\section{METHODS}

A cross sectional observational study was conducted from January 2019 to June 2019 with a questionnaire survey on 40 patients undergoing treatment with FFRD appliance (group 1,
20 patients) and Sharma's Class II corrector (group 2, 20 patients) assessing discomfort, expectations and experiences with the appliance. Sample size was calculated using formula for calculating difference between two proportions. Simple random sampling was done. Informed consent was obtained from the patients. The questionnaire was structured and validated, and reliability testing was done before the start of study. Questions were divided to evaluate aesthetics, functionality and comfort with the appliance. (Table 1 and 2). The statements (1, 3 and 9) evaluated the aesthetics of the appliance and statements $(2 \mathrm{~A}, \mathrm{~B}, 4,5,8,11)$ evaluated the changes in the normal functions of the oral cavity being performed with the appliance in place. The statements $(6,7$, $10,12-15)$ evaluated the comfort of wearing the appliance. The study design obtained ethical clearance from institutional ethics committee (DMIMS, DU). The data was collected by principal author in person from the patient in writing.

\section{Statistical Analysis}

Statistical analysis was done using SPSS (Statistical Package for Social Sciences) version 24.0 (IBM Corporation, Chicago, USA). Descriptive and analytical statistics were done. The difference in proportions was calculated by chi-square test. The Pearson's chi-square test with Fisher's exact test was used to discover if there is a relationship between two categorical variables. The level of significance was set at $\mathrm{P}<0.05$. (Table 1)

\section{RESULTS}

$30 \%$ of cases in group 2 indicated that the Sharma's Class II corrector looks good (Q1) as compared to $15 \%$ in group 1. (P $=0.630$ ) (Figure 1). $5 \%$ indicated it was not aesthetic in group 2 as compared to $10 \%$ in group 1 . For functional evaluation, questions regarding changes in speech, comfort in eating, swallowing and changes in sleeping pattern were asked.

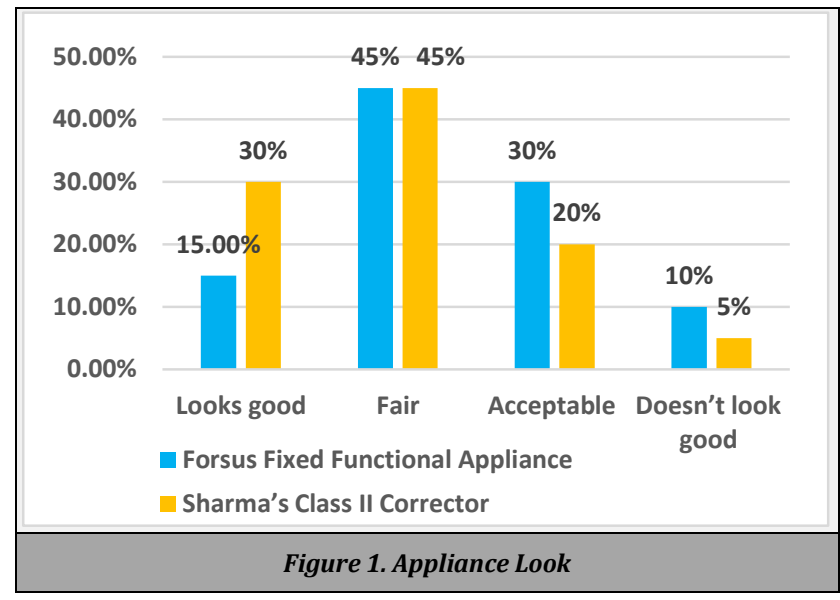

When patients were asked about comfort in speech with the appliance (Q2A), $40 \%$ patients in group 2 stated that it was not as affected and improved with time as compared to $0 \%$ in group 1. 


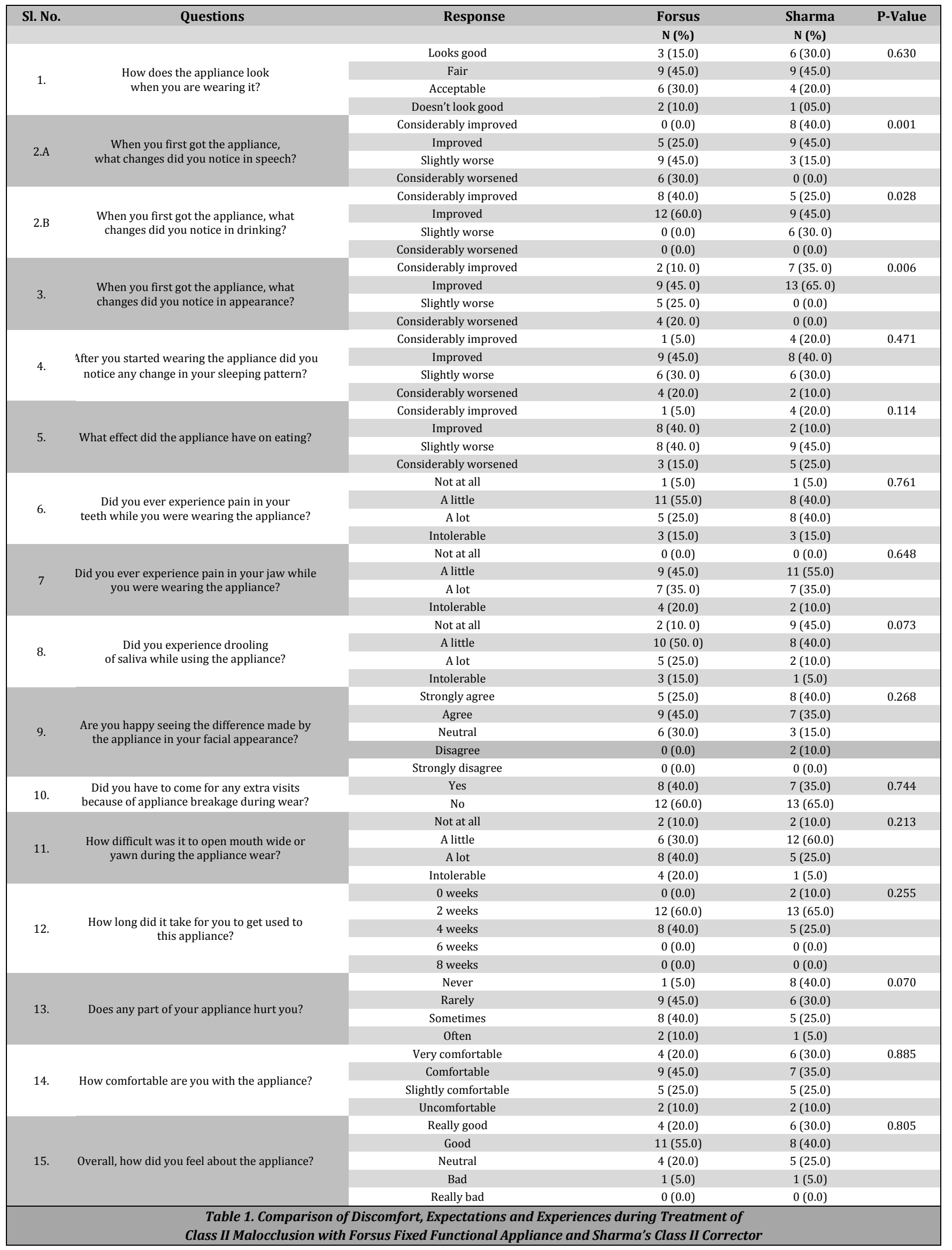



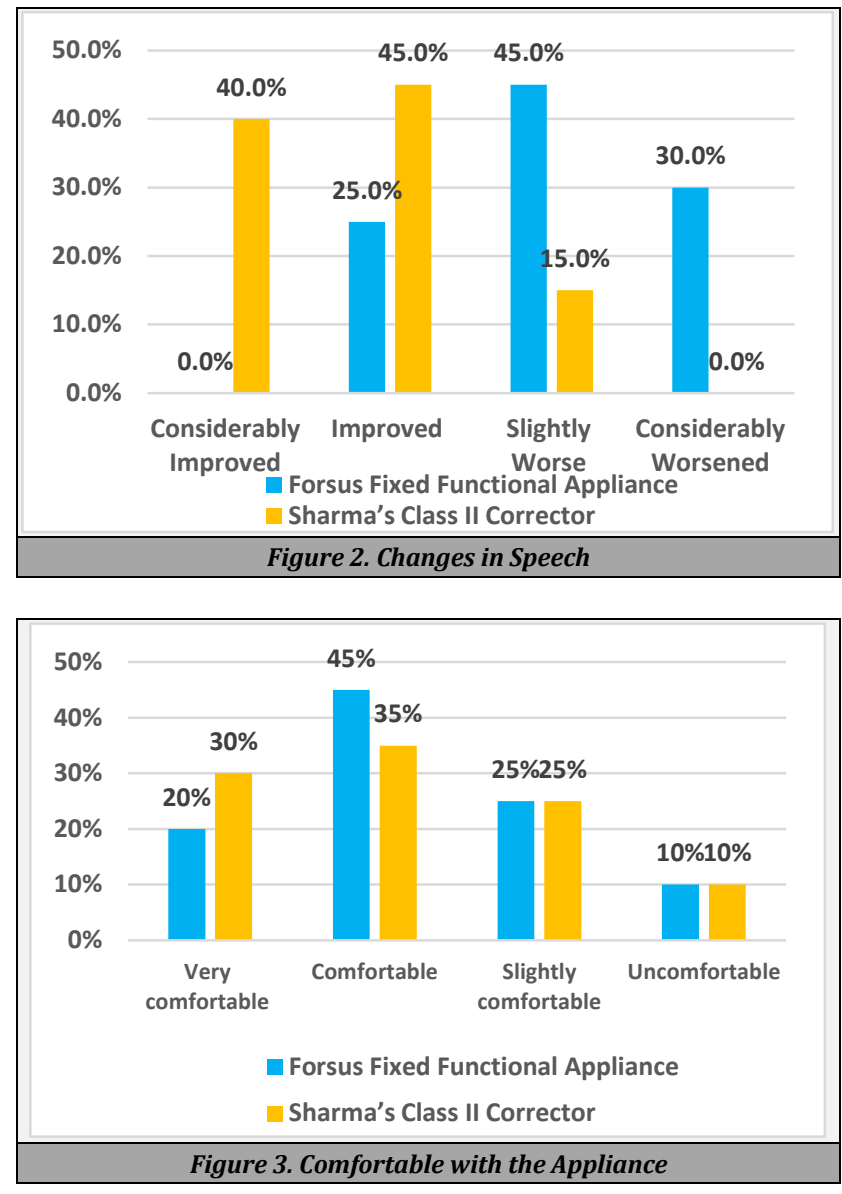

In group 1, $30 \%$ of cases indicated that there were problems associated with speech as compared to $0 \%$ or more in group 2. $(\mathrm{P}<0.05)$. Values were statistically significant. (Figure 2). Whereas comfort in swallowing improved in $40 \%$ of the patients in group 2 and $0 \%$ in group 1.12 out of 20 patients $(60 \%)$ stated that their sleeping pattern (Q 4,) improved after giving Sharma's Class II corrector appliance (group 2), whereas it was found to be improved only in $50 \%$ of group 1 patients. On the contrary it considerably worsened in 10 patients $(50 \%)$ in group $1(\mathrm{P}=0.471)$. No significant values were found on comparing eating and pain in the teeth and jaws with the use of the appliance in both the groups. (Q 5, $6,7)(P=0.114, P=0.761, P=0.648) .90 \%$ patients in group 1 complained of drooling of saliva (Q 8) while using the FFRD appliance (group 1). Whereas this reduced to $55 \%$ in patients using Sharma's Class II corrector appliance. $(\mathrm{P}=0.073)$

5 out of 20 patients ( $25 \%$ ) agreed that they are happy with the difference in their facial appearance after using FFRD, whereas this increased to $40 \%$ in patients using Sharma's Class II corrector. ( $\mathrm{Q} 9, \mathrm{P}=0.268) .40 \%$ of patients in group 1 had to come for extra visits because of appliance breakage during wear. This reduced to $35 \%$ in group 2, suggestive of lower incidence in breakage of Sharma's Class II corrector appliance. (Q 10, P = 0.744). Difficulty in wide mouth opening or yawning during the appliance wear was equally reported by both groups. ( $\mathrm{Q} 11, \mathrm{P}=0.213$ ). 2 patients in group 2 got used to the appliance in first 2 weeks itself, whereas no patients reported the same in group 1 . In both groups' patients were comfortable with the appliance within 4 weeks. (Q 12, P = 0.255). $40 \%$ of the patients in group 1 reported that sometimes the appliance had traumatised them. This reduced to $25 \%$ in group 2 patients. Also, in group 2, 8 patients reported that they never got traumatised by the appliance, but this count was of only 1 patient in group 1 . (Q $13, P=0.070)$. In both the groups patients were comfortable with the appliance but the values suggest that the comfort was slightly increased in group 2 as compared to group 1. (Q 14, 0.885) (Figure 3). When overall rating of the appliance was asked 20 $\%$ of the patients in group 1 rated it to be really good. This increased to $30 \%$ in group 2. (Q $15, P=0.805$ ).

\section{DISCUSSION}

A variety of skeletal as well as dental apparatus together comprise Class II malocclusion. One of the tests tested FFA used routinely is the FFRD, which has an advantage of its ability to be used in combination with comprehensive fixed appliance therapy. But it has the disadvantage of the tendency of its support system to break frequently, labial flaring of lower anterior. Also, this appliance is costly and not economical. If any part of the device breaks or is lost, a new appliance has to be procured which again compromises the cost effectiveness and time is lost till new appliance is received. To overcome the disadvantages of FFRD and to make it cost effective Sharma's Class II corrector was designed.

A research on comparison of before and after treatment results of Sharma's Class II corrector with FFRD in skeletal Class II division 1 malocclusion with retro positioned lower arch has shown considerable hard as well as soft tissue outcomes, stability of the effects of Sharma's Class II corrector, which are similar to skeletal, dental as well as soft tissue outcome of the FFRD.

A study was conducted in 2013 by Bowman et al. 6 to see patient's experience with FFRD. But there is lack of data about patient's perspective and experience with Sharma's Class II corrector appliance. Hence, a questionnaire survey was conducted to evaluate and compare discomfort, expectations and experiences during treatment of Class II malocclusion with Forsa's fixed functional appliance and Sharma's Class II corrector.

Q1, Q2a and Q2b evaluated look of the appliance, change in speech and changes in swallowing with the appliance respectively. It was found that all 3 patients improved in Sharma's Class II corrector as compared to patients treated with FFRD. When Bowman et al. evaluated the speech with FFRD, they stated that $2.9 \%$ of the patients responded as improved, whereas in our study $25 \%$ patients reported as improved. $45 \%$ patients wearing Sharma's Class II corrector responded that speech improved after wearing of the appliance. Hence, significant difference exists between the 2 groups. $(\mathrm{P}<0.05)$

In Q3. Result suggests, patients in Sharma's Class II corrector group noticed improved appearance after wearing the appliance as compared to FFRD group ( $\mathrm{P}=0.006) .27 .5 \%$ patients in a study ${ }^{6}$ reported that sleeping pattern worsened after wearing of FFRD appliance which was in accordance to our findings (30\%) and was similar to that of Sharma's Class II corrector group, when asked in Q4. (P = 0.471) Effect of appliance on eating showed no considerable difference in both groups. ( $\mathrm{P}=0.114)$

Bowman et al. found that $62.9 \%$ and $28.6 \%$ FFRD patients experience a little and a lot pain in teeth respectively, our 
study got the similar values of $55 \%$ and $25 \%$ in FFRD group. But it is interesting to note that pain in teeth after appliance wear decreased to $40 \%$ in patient's wearing Sharma's Class II corrector. (Q 6, P = 0.761)

Results of Q 8 suggest that the drooling of saliva is considerably lower in patient's wearing Sharma's Class II corrector as compared to FFRD group. ( $\mathrm{P}=0.073) 25 \%$ and $40 \%$ respectively. This may be attributed to the skeletonised design of Sharma's Class II corrector owing to which bulkiness of the appliance is reduced and it has increased flexibility.

Patients in both the groups were used to the appliance within 4 weeks and findings were in harmony with those reported by Tian XG et al. ${ }^{7}$ No significant results were found when extra visit for the appliance breakage was enquired. But Sharma's Class II corrector seem to hurt the patients less as compared to FFRD $(\mathrm{P}=0.070)$.

The difference made by the Sharma's Class II corrector in the Facial appearance was more accepted by the patients ( 40 $\%$ ). Equal number of patients in both the groups have rated the appliance from slightly comfortable to very comfortable $(90$ $\%$ ), whereas $10 \%$ patients in both groups found the appliance to be uncomfortable.

Tian XG et al. ${ }^{7}$ reported (83.7\%) neutral to favourable experience with Forsus. It was slightly more in our study i.e. $95 \%$ patients agreed it being neutral to really good. And equal result was seen with patients using Sharma's Class II corrector. Common cost of the treatment for both the groups included Upper and lower working model impressions, molar bands and cementation and fixed orthodontic. The only difference was in the cost of FFA. FFRD appliance costs 6800 Rs. to which cost of breakages (if any) was added. Whereas cost of Sharma's Class II corrector is no more than 150 Rs. The reason being it is made of simple wire bending of a readily available orthodontic wire. Thus, Sharma's Class II corrector is cost effective as compared to FFRD.

\section{CONCLUSIONS}

Sharma's Class II corrector has similar patient acceptance as compared to FFRD appliance with the additional benefit of cost effectiveness. Hence this can be considered as a better option in treating Class II malocclusion with fixed therapy.

Data sharing statement provided by the authors is available with the full text of this article at jemds.com.

Financial or other competing interests: None.

Disclosure forms provided by the authors are available with the full text of this article at jemds.com.

\section{REFERENCES}

[1] Zymperdikas VF, Koretsi V, Papageorgiou SN, et al. Treatment effects of fixed functional appliances in patients with Class II malocclusion: a systematic review and meta-analysis. Eur J Orthod 2016;38(2):113-26.

[2] Karia H, Shrivastav S, Karia AK. Three-dimensional evaluation of the airway spaces in patients with and without cleft lip and palate: a digital volume tomographic study. Am J Orthod Dentofacial Orthop 2017;152(3):37181.

[3] Kamble R, Sharma NS, Shrivastav S, et al. A tailored approach for growth modification: an innovative approach. World Journal of Dentistry 2017;8(4):334-42.

[4] Vogt W. The forsus fatigue resistant device. J Clin Orthod 2006;40(6):368-77.

[5] Sharma NS, Sharma P, Shrivastav S, et al. Sharma's bite corrector appliance. The Journal of Indian Orthodontic Society 2014;48(8):573-7.

[6] Bowman AC, Saltaji H, Flores-Mir C, et al. Patient experiences with the forsus fatigue resistant device. Angle Orthod 2013;83(3):437-46.

[7] Tian XG, Wu ZZ, Zhang SJ, et al. Patients' experiences with the Forsus appliance. Shanghai Kou Qiang Yi Xue 2014;23(2):209-13. 